\title{
Extraction from Tev-Range Accelerators using Bent Crystal Channeling
}

\author{
R.A. Carrigan, Jr., Dong Chen, P. Colestock, D. Herrup, G.Goderre, \\ G. Jackson, C.T. Murphy and R. Stefanski \\ Fermi National Accelerator Laboratory, P.O. Box 500, Batavia, Illinois 60510
}

S. Baker, N. Mokhov, B. Parker, H.-J. Shih, R. Soundranayagam and T. Toohig

Superconducting Super Collider Laboratory, Dallas, Texas 75237

S. Peggs

Brookhaven National Laboratory, Upton, Long Island, New York 11973

C.R. Sun

State University of New York at Albany, Albany, New York 12222

A. Boden, D. Cline, W. Gabella, S. Ramachandran, J. Rhoades and J. Rosenzweig

University of California at Los Angeles, Los Angeles, California 90024

M. Arenton, S. Conetti, B. Cox, C. Dukes, V. Golovatyuk, A. McManus, K. Nelson and B. Norem The University of Virginia, Charlottesville, Virginia 22901

B.Newberger

The University of Texas, Austin, Texas 78712

J.A. Ellison

The University of New Mexico, Albuquerque, New Mexico 87131

A. Erwin

The University of Wisconsin, Madison, Wisconsin 53706

R. Rossmanith

CEBAF, New port News, Virginia 23606

A. Kovalenko, A. Taratin, N. Malakhov and E. Tsyganov Joint Institute for Nuclear Research, Dubna, Russia

M. Bavizhev, V. Biryukov and M. Maslov

Institute for High Energy Physics, Serpukhov, Russia

A. Khanzadeev, T. Prokofieva, V. Samsonov and G. Solodov

Petersburg Nuclear Physics Institute, Gatchina, Russia

March 1994

Accepted in Nuclear Instruments and Methods

Operated by Universities Research Association Inc. under Contract No. DE-AC02-76CHO3000 with the United States Department of Energy 


\section{Disclaimer}

This report was prepared as an account of work sponsored by an agency of the United States Government. Neither the United States Government nor any agency thereof, nor any of their employees, makes any warranty, express or implied, or assumes any legal liability or responsibility for the accuracy, completeness, or usefulness of any information, apparatus, product, or process disclosed, or represents that its use would not infringe privately owned rights. Reference herein to any specific commercial product, process, or service by trade name, trademark, manufacturer, or otherwise, does not necessarily constitute or imply its endorsement, recommendation, or favoring by the United States Government or any agency thereof. The views and opinions of authors expressed herein do not necessarily state or reflect those of the United States Government or any agency thereof. 


\section{EXTRACTION FROM TEV-RANGE ACCELERATORS USING BENT CRYSTAL CHANNELING}

R. A. Carrigan, Jr., Dong Chen, P. Colestock, D. Herrup, G. Goderre, G. Jackson, C. T. Murphy, and R. Stefanski Fermi National Accelerator Laboratory, Batavia, Illinois 60510, USA

S. Baker, N. Mokhov, B. Parker, H.-J. Shih, R. Soundranayagam, and $T$. Toohig

Superconducting Super Collider Laboratory, Dallas, TX 75237, USA

S. Peggs

Brookhaven National Laboratory, Upton, LI, NY 11973, USA

C. R. Sun

State University of New York at Albany, Albany, NY 12222, USA

A. Boden, D. Cline, w. Gabella, S. Ramachandran, J. Rhoades, and J. Rosenzweig

University of California at Los Angeles, Los Angeles, CA 90024, USA

M. Arenton, S. Conetti, B. Cox, C. Dukes, V. Golovatyuk, A. McManus, K. Nelson, and B. Norem

The University of Virginia, Charlottesville, VA 22901, USA

B. Newberger

The University of Texas, Austin, TX 78712, USA

J. A. Ellison

The University of New Mexico, Albuquerque, NM 87131, USA

A. Erwin

The University of Wisconsin, Madison, WI 53706, USA

R. Rossmanith

CEBAF, Newport News, VA 23606, USA

A. Kovalenko, A. Taratin, N. Malakhov, and E. Tsyganov Joint Institute for Nuclear Research, Dubna, Russia

M. Bavizhev, V. Biryukov, and M. Maslov

Institute for High Energy Physics, Serpukhov, Russia

A. Khanzadeev, T. Prokofieva, V. Samsonov, and G. Solodov

Petersburg Nuclear Physics Institute, Gatchina, Russia 


\title{
Abstract
}

\begin{abstract}
Plans and first results from Fermilab Experiment 853 are presented. E853 is an experiment to test the feasibility and efficiency of extracting a low-intensity beam from the halo of the Tevatron using channeling in a bent silicon crystal. The motivation of the experiment is to apply crystal extraction to trans-TeV accelerators like the LHC. Channeling developments related to crystal extraction and some early results from accelerator studies at the Tevatron are presented.
\end{abstract}

\section{Introduction}

The possibility of beam extraction from accelerators using bent crystals has been discussed since Tsyganov first proposed bent crystal channeling ${ }^{1}$. Protons have already been extracted using bent crystals at Dubna ${ }^{2}$, Serpukhov ${ }^{3}$, and the SPS at CERN ${ }^{4}$. The idea of extracting halo beam from the SSC with a bent crystal was first seriously discussed by $C$. $R$. Sun ${ }^{5}$. Further considerations of the idea $6,7,8$ led to a proposal ${ }^{9}$ that the SSC (Superconducting Super Collider) East Campus footprint be modified to make this possibility feasible at a later time. A proposal for an experiment which would use such a beam to do fixed-target B-physics was submitted to the SSC ${ }^{10}$. The possibility of an SSC facility led to a proposal for an experimental feasibility study of this extraction method in the Fermilab Tevatron, a superconducting accelerator similar to the SSC and the LHC. This experiment (E853) has now been approved for 72 hours of dedicated study time during the Fermilab Collider Run in 1994. Some of the accelerator tests connected with the experiment have already been carried out.

The experiment and the associated channeling studies have several goals. For channeling the crystal must be able to be aligned to the beam quickly, crystal quality must be satisfactory, and the crystal must be able to survive the radiation damage due to the proton beam. One goal of the accelerator experiment is to extract one million $900 \mathrm{GeV} / \mathrm{c}$ protons/s with $10^{12}$ protons circulating. Other goals are to show that the luminosity lifetime is not seriously shortened and that no intolerable backgrounds are created at the Tevatron collider experiments. In addition, the relationship between the RF (radio frequency) modulation amplitude used for extraction and the extraction efficiency will be determined. 


\section{Trans-TeV Extraction}

The idea of extracting the natural halo of the circulating SSC and LHC beams to make low-intensity beams has intrigued people for the last decade. Since this halo will eventually be absorbed on "scraper" collimators, why not put it to better use? The proposal for the SSC was to install a copy of the abort insert planned for the SSC West Utility straight Section in the unused East utility straight section, but with a bent crystal replacing the abort kicker magnets. A crystal bent by 160 microradians would channel halo beam incident on it up into the field-free hole of a string of Lambertson magnets. (A Lambertson magnet contains a field-free region separated from the normal magnetic region by a several $\mathrm{mm}$ septum.) From there, the beam would exit the accelerator and enter a hall for detectors $2200 \mathrm{~m}$ downstream, separated laterally from the accelerator by $60 \mathrm{~m}$. Details of the SSC extraction concept and Monte Carlo simulations of it are given in an earlier paper and the references therein.

\section{The Tevatron Experiment}

E-853 is taking place in the co straight section of the Tevatron, the normal location of the proton abort line. The abort line consists of a three-bend magnetic dogleg that provides a 4 mrad horizontal kick so the abort line can clear the magnets at the downstream end of the long straight section. The middle bend in the dogleg consists of a series of Lambertson magnets. During collider runs, the abort line is not used at $900 \mathrm{GeV}$, so one of the kicker magnets has been replaced by a bent crystal. Figure 1 shows the location of the crystal in the $C 0$ region. As shown in Figure 2 , the crystal is positioned to the side of the beam with an upward curvature of 640 prad. This is sufficient to deflect the beam halo that strikes the crystal into the field-free region of the Lambertson magnets where the particles can continue rising.

The crystal is mounted at the upstream end of a 1-m beam pipe which ends with articulating bellows. Two precision motors ( $x, y)$ at each end of the pipe allow for the alignment of the crystal with four degrees of freedom. The most critical parameter is the alignment of the vertical angle of the crystal with the beam angle, which must be done to within $10 \mu \mathrm{rad}$ in order to match the critical angle of the crystal $(5.2 \mu \mathrm{rad}$ at $900 \mathrm{GeV})$ with the beam vertical angular divergence of 11.5 prad.

There are two air gaps in the extracted particle line separated by $40 \mathrm{~m}$ in which there will be four scintillators and four silicon strip planes to count the extracted beam and measure its trajectory precisely. This instrumentation is used to prove that the extracted beam is channeled beam and not just scattered background. Since the $\mathrm{CO}$ abort line is used for disposing of 150 GeV protons during Tevatron injection the detectors in the line 
must retract when the Tevatron is not in a $900 \mathrm{GeV}$ store. This is accomplished with horizontal motion stages driven by standard stepping motors.

\section{Channeling Considerations}

Our recent studies ${ }^{12}$ indicate that for a crystal used for SSC or LHC extraction the number of type A dislocation loops should be kept small and that linear dislocations densities must be less than $1 / \mathrm{cm}^{2}$. The silicon crystal to be used for Tevatron extraction has been selected to be dislocation-free (less than 1 dislocation $/ \mathrm{cm}^{2}$ ). A suitable sample was found by observing the line width in double $x$-ray scattering and by using film decoration techniques ${ }^{13}$. The 40 $\mathrm{mm}$ long crystal is $10 \mathrm{~mm}$ wide and $3 \mathrm{~mm}$ thick so that it is substantially thicker than the vertical beam diameter $\left(\sigma_{\mathrm{v}}=0.32 \mathrm{~mm}\right)$. With the use of $x$-ray scattering the crystal has been oriented so that the curved surface contains a (110) plane that will be parallel to the accelerator beam at the upstream end of the crystal. The techniques used for this crystal analysis are described in more detail in other publications ${ }^{13}$.

The alignment and flatness of the vertical surface facing the circulating beam (the effective septum face) are critical factors. These factors must be handled carefully to minimize the thickness of the region for which an incident proton would go in and out of the crystal and be only partially bent. A particle must strike deep enough into the crystal so that it sees an uninterrupted lattice structure. It is certainly possible to achieve a flatness of 0.5 microns (the wavelength of light), but it is hoped to do substantially better, as the typical step size for the beam at extraction will be on the order of $1 \mu \mathrm{m}$.

The 640 urad bend angle of the crystal must be controlled to $120 \mu \mathrm{rad}$, half the acceptance angle of the extraction channel. The sagitta of the $20 \mathrm{~mm}$ bent part of the $40 \mathrm{~mm}$ crystal is $1.6 \mu \mathrm{m}$, measurable only by high-precision interferometric methods. An analysis $^{14}$ of the effect of the holder on the crystal lattice was carried out with the finite element program ANSYS to simulate the stresses and deformation in the crystal while being squeezed in the holder. It was found that due to the finite stiffness of the aluminum benders and the flap-back of the bent crystal, a design bend for the aluminum bender of $0.96 \mathrm{mrad}$ was required to get an actual full bending angle of $0.64 \mathrm{mrad}$ in the crystal. Along the surface of the crystal facing the beam, the variation of this bend angle was negligible at the entrance and exit of the crystal due to the silicon overhang beyond the lengths of the aluminum pieces. The force required to accomplish this bend is less than $5 \mathrm{Kg}$ with a maximum stress in the crystal of less than $10^{7}$ pascals. This same study shows that at the crystal entrance the top edge of the surface of the crystal facing the beam is closer to the beam than the bottom edge by $0.04 \mu \mathrm{m}$. Because this distortion is small this 
effect is not expected to affect the channeling efficiency.

The possibility of radiation damage of the crystal has been investigated. In a study at $\mathrm{BNL}^{15}$ at high fluence we have found measurable radiation-induced dechanneling produced at a fluence of $4 * 10^{20}$ protons $/ \mathrm{cm}^{2}$. While this is of some concern at the beam intensities expected for the LHC, particularly for radiationinduced dislocations ${ }^{12}$, it is not significant for the lower beam intensities and short runs planned for the Tevatron tests. Heating effects of the beam losses on the crystal have also been calculated and are negligible at the Tevatron.

\section{Slow Parasitic Extraction Driven by RF Noise}

The key to parasitic slow extraction at high energy is to inject noise into the accelerator RF system. This pumps beam from the outer edge of the core of the beam into the horizontal halo where it will eventually intercept the crystal on some turn following the appropriate number of betatron oscillations. The extraction process must be very efficient in order to provide the greatest flux possible to a future fixed target experiment while simultaneously maximizing the proton intensity and luminosity lifetimes during each store. In addition the process cannot produce significant background at a collider experiment. Finally, because the fixed target experiments will be rate-limited to no more than one proton passage per RF bucket, a slow and steady method of extraction is necessary.

The challenge is that there is inadequate natural halo to obtain extracted intensities high enough to be interesting for experiments. Halo must be generated by perturbations of either the transverse or longitudinal phase space in a manner which does not appreciably decrease the collider luminosity. As a result, the usual method of resonant extraction in the horizontal plane is not permitted. For that reason, techniques have been investigated that create off-momentum halo in longitudinal phase space using RF voltage modulations and thereby continuously populate the region of phase space near the crystal. The crystal is placed at a point of high dispersion so that the off-momentum particles are at large $x$ at the crystal as illustrated schematically by the ellipse in Figure 2 .

In this approach particles which are already in the tail of the momentum distribution are rapidly excited to larger momenta so as to achieve large step sizes, without affecting very much the core of the momentum distribution. The most promising technique of populating the halo (the CERN ${ }^{16}$ approach is along the same line) is by generating amplitude-dependent diffusion rates in either the longitudinal (SSC and Tevatron) or transverse (LHC and SPS) planes. By generating a signal which has a small effect at low amplitudes but generates large particle diffusion rates at greater oscillation 
amplitudes, luminosity lifetime can be preserved while creating a steady state population of particles which feed into the crystal. These are observed in Monte Carlo simulations to strike well into the crystal (>1 $1 \mathrm{~m}$ ) with the betatron motion aiding the penetration. This avoids surface irregularities and crystal edge misalignments and maximizes the extraction efficiency. This diffusion rate profile is generated by taking advantage of phase space non-linearities which create amplitude-dependent particle tunes. Since each particle reacts only to RF signals at their local resonant frequencies, frequency-dependent signal power densities cause amplitude dependent diffusion rates. Though in most cases simply-shaped random $R F$ noise is utilized, more complicated waveforms have also been investigated as a mechanism to improve the mean penetration depth into the crystal ${ }^{17}$.

A diffusion model ${ }^{18}$ has been developed for crystal extraction using RF noise-induced halo growth based on a diffusion equation. This has some similarities to the diffusion in transverse energy approach used for studying crystal dechanneling. Monte Carlo simulations ( 1000 particles) have also been used to track diffusing particles through a million turns of the SSC lattice. The diffusion results (which are less computationally-intensive) and the simulation program agree. The simulation shows that there are viable scenarios (for both transverse and longitudinal growth) to provide halos without disturbing the core.

We have also investigated a second approach to increasing the penetration depth into the crystal ${ }^{19}$ by adding another thin, aligned crystal to spread the beam with channeling oscillations. This could increase the penetration into the bent crystal substantially and relax the radiation load on the crystal.

\section{Early Results Related to TeV-Range Extraction}

During the recent Tevatron collider run, an unbent crystal was placed at the planned location of the bent crystal but to the outside of the ring. This was used to study whether halo beam scattered by the crystal created intolerable backgrounds at either of the two collider experiments ${ }^{20}$. Several sets of measurements were performed. The effect of RF noise on the beam in the absence of collimation was studied during a store at $900 \mathrm{GeV}$. Collimation effects were also observed with conventional collimators and the silicon crystal at the proposed bent crystal location.

For the diffusion studies two levels of external random noise were applied to the RF system. With an rms external voltage of 500 $\mathrm{mV}$, corresponding to an rms RF gradient fluctuation of $5 \mathrm{kV} / \mathrm{turn} i t$ was found that the longitudinal density narrowed while there were many more particles at large amplitude. Once the equilibrium shape of the longitudinal bunch distribution was established, an exponential particle loss rate appeared. With $5 \mathrm{KV} /$ turn noise, the 
relative proton loss rate corresponded to a beam lifetime loss constant of 12 minutes. With a reduced noise level of $50 \mathrm{mV}$ (an RF voltage jitter of $500 \mathrm{~V}$ rms) the loss rate time constant was 17 hours, so that a factor of 10 reduction in noise amplitude was responsible for a 100-fold loss rate reduction. The nominal intensity time constant for Tevatron Collider protons varies from 40 to 120 hours.

To estimate the impact of a bent crystal on the CDF (Collider Detector at Fermilab) detector a horizontal collimator was placed next to the beam at $A 0$, one-third around the ring from $C 0$. The collimator was brought in until losses were observed on it. The rms RF amplitude noise level was set at $500 \mathrm{~V} / \mathrm{turn}$. Even though this noise level induced a loss rate ten times that which is desired for crystal extraction, the maximum proton background rate measured in the CDF detector was $5 \mathrm{KHz}$. Depending on the luminosi$t_{Y}$, a background rate below the 5-10 $\mathrm{kHz}$ range is considered acceptable at the CDF detector.

In order to assure that the measurements made with the collimator were meaningful for crystal extraction calculations, an unbent silicon crystal was installed on the radial outside of the accelerator beam, so that it could not intercept DC beam. (DC beam consists of those particles which have diffused out of the RF bucket and are spiraling radially inward due to synchrotron radiation losses.) on the other hand, particles with large betatron amplitudes could strike the silicon crystal as their momentum error increased. With the crystal as the primary aperture and the same diffusion conditions as above ( 10 times that planned for extraction), it was found that the CDF loss increased from approximately $2 \mathrm{KHz}$ to $10-15 \mathrm{KHz}$.

Based on these studies, the effects of crystal extraction should have little or no deleterious effects on a collider experiment and it should be possible to perform parasitic studies of crystal extraction during a collider run.

A group at CERN is currently carrying out a similar experiment $^{16}$ in the SPS, operating at $120 \mathrm{GeV}$. Their method of inducing diffusion is to introduce white noise on a horizontal damper (electrostatic plates capable of deflecting the beam a few tens of urad). To date, they report extracting beam with an efficiency of about 98. Their studies indicate that it is important to consider multi-turn extraction, since a particle first incident on the crystal with an angle greater than the critical angle will be multiply-scattered by the crystal to a different point in phase space and often will reenter the crystal on a later betatron oscillation with a smaller angle. 
Acknowledgements :

We would like to acknowledge the support of the SSC Laboratory for the equipment for this experiment, and the support of the Fermilab Accelerator Division in the early installation work. This work was supported in part by the US Department of Energy under contracts DE-AC35-89-ER-40486 and DE-AC02-76CH0300.

Figure Captions:

1. Schematic of the beam line around the Tevatron Co region; the crystal bends up out of the page into the field-free region of the Lambertson magnet chain.

2. View of the circulating beam looking downstream at the bent crystal. The ellipse represents the halo of the beam schematically. The crystal is bent upwards $640 \mu \mathrm{rad}$ and is on the inside of the ring. The parallel lines represent the crystal planes. 


\section{References :}

1. E. N. Tsyganov, Fermilab TM-682 (1976).

2. V. V. Avdeichikov et al., JINR 1-84, Dubna (1984). English translation: Fermilab FN-429 (1986).

3. See, for example, A. A. Asseev, M. Bavizhev, E. A. Ludmirsky, V. A. Maisheev, and Yu. S. Fedotov, Nucl. Instr. and Meth. A309, 1 (1991).

4. H. Akbari, et al., Phys. Lett. B313, 491 (1993).

5. C. R. Sun, R. A. Carrigan, Jr., T. E. Toohig, and D. Neuffer, in Proceedings of the Summer Study on the Design and utilization of the SSC (Snowmass), eds. R. Donaldson and J. Morfin, p. 483 (APS, New York, 1984).

6. M. Harrison and T. Toohig, in Proceedings of the 1986 Summer Study on the Physics of the SSC (Snowmass), eds. R. Donaldson and J. Marx, p. 534 (APS, New York, 1986).

7. A. S. Vodopyanov et al., Slow Extraction of $20 \mathrm{TeV}$ Accelerated Bean out of SSC Using a Bent Crystal, preprint of the Joint Institute for Nuclear Research, Dubna (1987).

8. B. Cox et. al., in Proceedings of the Summer Study on High Energy Physics in the 1990's (Snowmass), ed. S. Jenson, p. 538 (World Scientific, New Jersey, 1988).

9. C. T. Murphy and R. Stefanski, SSCL Note 428 (1989).

10. The SFT Collaboration, An Expression of Interest in a Super Fixed Target B Physics Facility at the SSC, EOI-14, submitted to the SSC Laboratory $(1990)$.

11. H. Brown et. al., in Proceedings of the 1990 Summer Study on High Energy Physics (Snowmass), ed. E. Berger, p. 373 (World Scientific, New Jersey, $1990)$.

12. V. M. Biryukov, M. D. Bavizhev, and E. N. Tsyganov, SSCL-N-777 (1991).

13. V. V. Baublis et al., to be published in Nucl. Instr. and Meth. (1993).

14. z. Tang, "Silicon Crystal Under Bending", Fermilab TM-1827 (1993).

15. S. Baker et al., NIM to be published (1993).

16. S. Weisz, et al., "Proton Extraction from the CERN SPS by a Bent Crystal", Proc. 1993 Part. ACC. Conf., washington, DC (1993). Status Report on RD22: Crystal Extraction at the SPS", CERN Report CERN/DRDC 92-51 (1992).

17. W. Gabella, J. Rosenzweig, R. Kick, and S. Peggs, "RF Voltage Modulation at Discrete Frequencies, with Applications to Crystal Channeling Extraction", Proc. 1993 Part. ACC. Conf., Washington D.C. (1993). 
18. B. S. Newberger, H.-J. Shih, and J. A. Ellison, Nucl. Instr. and Meth. A235, 9 (1993). B. S. Newberger, H.-J. Shih, and J. A. Ellison, Phys. Rev. Lett. 71, 356 (1993).

19. A. Taratin, E. Tsyganov, M. Bavizhev, A. Chao, H.-J. Shih, and T. Toohig, SSCL-545 (1991).

20. G. Jackson, "Results from Beam Diffusion and Collimation Measurements in Preparation for Fermilab Tevatron Crystal Extraction", Proc. 1993 Part. Acc. Conf., Washington D.C. (1993). 


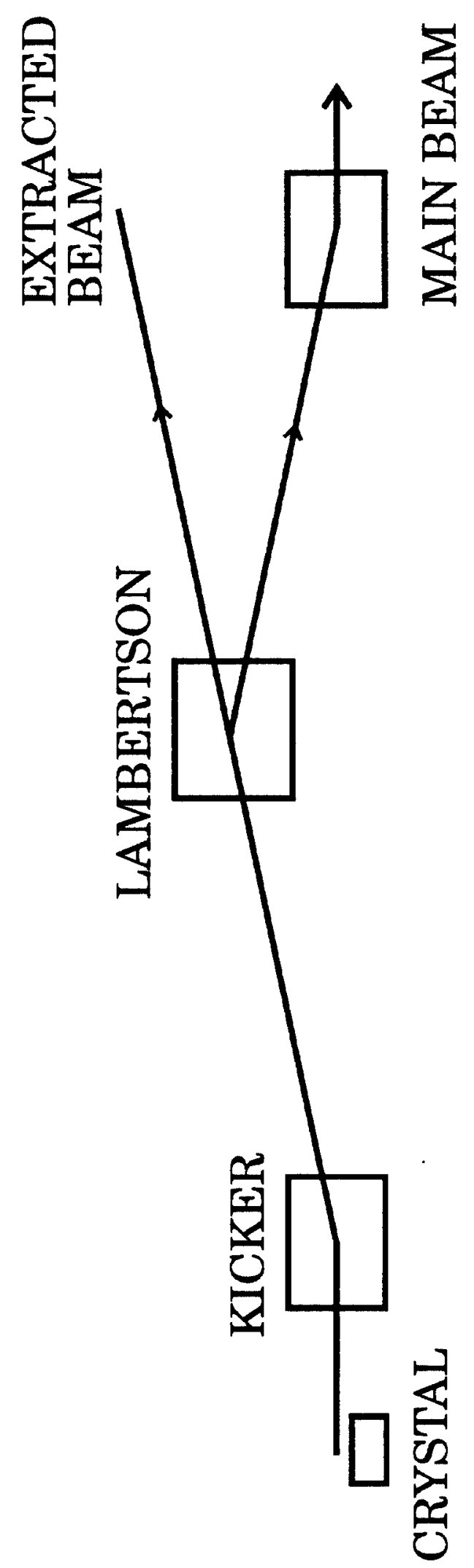



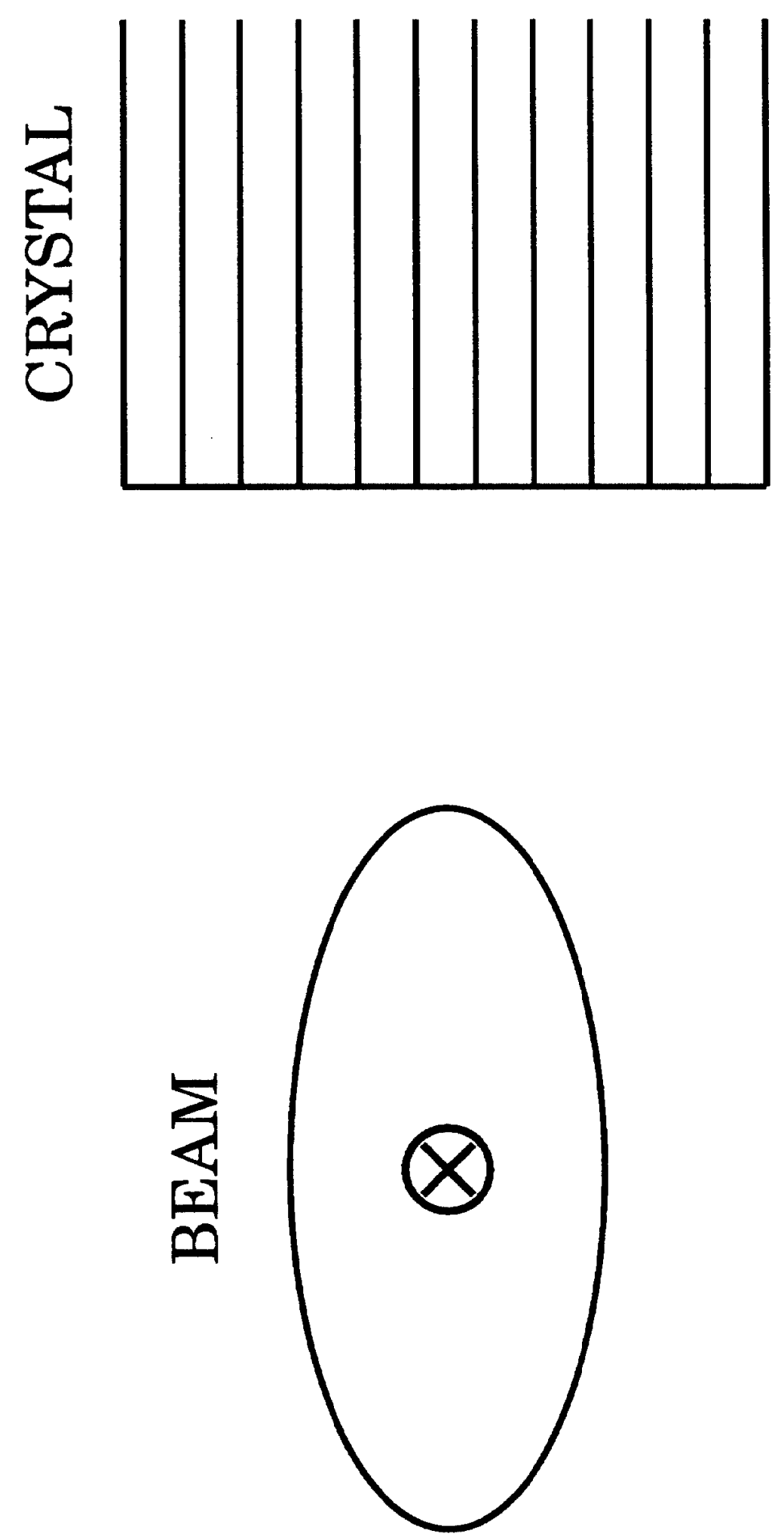